\title{
SUCCESS OF INTRAVITREAL RANIBIZUMAB INJECTION IN ACUTE CENTRAL SEROUS CHORIORETINOPATHY
}

\author{
Chalid Kurniawan ${ }^{1,2}$, Arief S Kartasasmita ${ }^{1,3}$ \\ 'Santosa Eye Center of Santosa Hospital Bandung Central, \\ ${ }^{2}$ Department of Ophthalmology Faculty of Medicine Universitas Pasundan, \\ ${ }^{3}$ Department of Ophthalmology Faculty of Medicine Universitas Padjadjaran
}

ABSTRACT

\begin{abstract}
Introduction: Central serous chorioretinopathy (CSC) manifests as neurosensory detachment of the macula and observation has been considered as an appropriate first-line approach. Active treatment is required to prevent recurrences or chronic type. This study aim to evaluate the effectiveness of intravitreal ranibizumab injection (IVRI) for acute CSC.
\end{abstract}

Methods: A single-center retrospective descriptive study. Five files of the patients who had the diagnosis of CSC then treated with IVRI between 2018 and 2019 have been investigated. Demographic details, bestcorrected visual acuity (BCVA), and central macular thickness (CMT) were studied before and after treatment.

Result: The mean age of the patients was 57 years ( $S D=10.5$ years) mostly female ( 3 patients). The mean duration before treatment was 3.8 weeks ( $S D=1.4$ weeks). The mean number of IVRI was $1.4(S D=0.5)$. The mean CMT before IVRI was $436.4 \mu \mathrm{m}(\mathrm{SD}=109.1)$ and decreased to $243 \mu \mathrm{m}(\mathrm{SD}=38.3)$ at the last visit. The mean BCVA before IVRI was $0.56 \log M A R(S D=0.37)$ and improved to $0.22 \log M A R(S D=0.29)$

Conclusion: IVRI appeared to be effective treatment for acute CSC. The mean CMT decreased and BCVA improved after therapy.

Keywords: central serous chorioretinopathy, intravitreal ranibizumab, central macular thickness

Cite This Article: KURNIAWAN, chalid. SUCCESS OF INTRAVITREAL RANIBIZUMAB IN ACUTE CENTRAL SEROUS CHORIORETINOPATHY. International Journal of Retina, [S.I.], v. 3, n. 2, sep. 2020. ISSN 2614-8536. Available at: https://www.ijretina.com/index.php/ijretina/article/view/106

*Correspondence to: ChalidKurniawan,

Department of Ophthalmology UniversitasPasundan,

chal_4@yahoo.com

\section{INTRODUCTION}

Central serous chorioretinopathy (CSC) is a retinal disorder characterized by a serous detachment of the neurosensory retina (SRD) and/or a detachment of the retinal pigmen epithelium (RPE) at the posterior pole. CSC affects young and middle-aged adults complaining blurred vision with a relative central scotoma and metamorphopsia associated with dyschromatopsia, micropsia, and a reduction in contrast sensitivity. ${ }^{1,2,3}$

Acute CSC usually resolves spontaneously within a few months with complete reabsorption of the fluid collection and visual acuity recovery. Yet a single episode of acute CSC may convert to multiple recurrences of SRD or result in chronic SRD with persistent fluid. Patients with acute CSC often desire more expedient resolution of their disease. Thus, other treatment option must reconsider than observation approach alone., ${ }^{1,2}$ Different treatment options are available including observation, photodynamic therapy (PDT) with vertaporfin, laser photocoagulation, pharmacotherapy, micropulse diode laser and 
intravitreal anti VEGF agents have been used. None of these therapy are the mainstay treatment of $\mathrm{CSC}^{1}{ }^{1,2,3,4}$

Ranibizumab as one of the anti-vascular endothelial growth factors (VEGF) drugs have been employed in the treatment of acute and chronic CSC. Ranibizumab was suggested to reducing choroidal vascular hyperpermeability, even intraocular concentration of VEGF levels is not increased. ${ }^{5,6,7}$ The aim of this study is to evaluate the effectiveness of intravitreal ranibizumab injection (IVRI) for acute CSC

\section{METHODS}

This study is a retrospective descriptive carried out in the Eye Center of Santosa Hospital Bandung Central. Data were collected from medical records and five files patients with acute CSC and treated with IVRI in the period 2018 until 2019 were analyzed. Informed consent had been signed before the treatment. The inclusion criteria were all patients with CSC diagnose for the first time, never had therapy for the CSC, documented neurosensory detachment on optical coherence tomography (OCT), and had follow up data at least one month after IVRI therapy. Patients with incomplete medical record data, had other disease in the retina, had undergone previous treatment with laser photocoagulation, photodynamic therapy (PDT), intravitreal triamcinolone, or bevacizumab in the previous 3 months were excluded. Base line characteristic of the patients, best-corrected visual acuity (BCVA) in logarithm of the minimal angle of resolution (logMAR) scale, and central macular thickness (CMT) were evaluated before and one month after treatment. At baseline and follow-up visits, intraocular pressure, dilated fundus examination, and OCT for measurement of CMT were recorded. All eyes were injected with intravitreal ranibizumab $(0.5 \mathrm{mg} / 0.05 \mathrm{ml})$.

BCVA is obtained with the best glasses correction in logMAR scale. CMT is the average thickness in the area of $500 \mu \mathrm{m}$ from the central macula obtained from OCT examination in $\mu \mathrm{m}$ units. The BCVA and CMT measurement was taken before injection and one month after injection.

\section{RESULTS}

Over period of 2 years there were five eyes from five patients included in this study. Baseline characteristic data for all patients are showed in table 1 .

Table 1. Baseline characteristics of Patients

\begin{tabular}{lc}
\hline \multicolumn{1}{c}{ Characteristics } & Patients and Eye \\
\hline Mean age (range), yrs & $57(40-69)$ \\
Gender, no of patients (\%) & $2(40 \%)$ \\
Male & $3(60 \%)$ \\
Female & $2(40 \%)$ \\
Lateralisation, no of eye (\%) & $3(60 \%)$ \\
$\quad$ Right eye & $3.8(2-6)$ \\
Left eye & \\
CSC duration weeks, mean (range) & \\
Presenting symptoms, no of patients & \\
(\%) & \\
Scotoma & $4(80 \%)$ \\
Metamorphopsia & \\
yrs=years, CSC=central serous chorioretinopathy
\end{tabular}

Most of the patients were female ( 3 vs 2 patients; 60\%). The average age is 57 years old with age range 40 to 69 years old. Metamorphopsia was the most symptoms complaining by the patients during their 2 to 6 weeks duration of CSC.

Improvement of visual acuity were achieved in 4 patients while 1 patient still had same visual acuity. Visual acuity before injection and 1 or 2 month after injection were shown in figure 1.

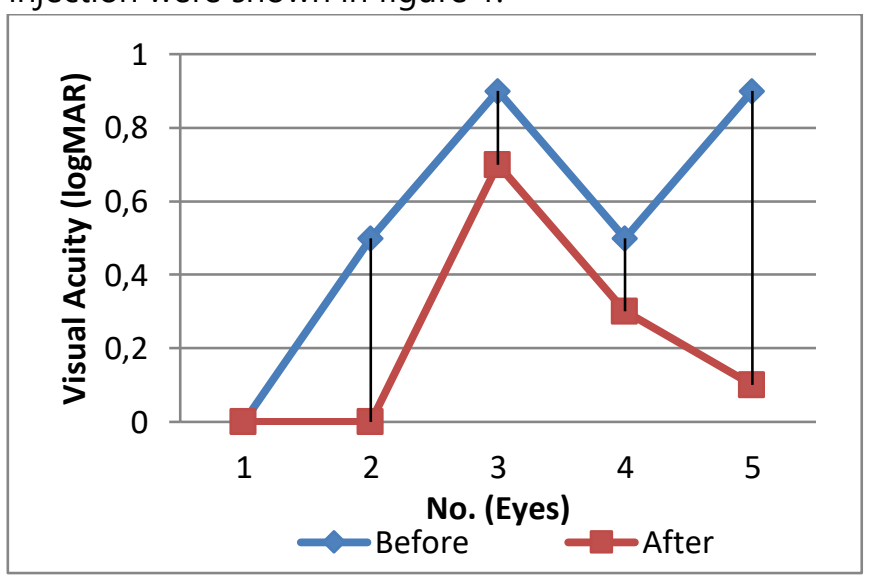

Figure 1. Visual Acuity Before and After IVRI

At the end of therapy there were decreasing in CMT from 5 patients undergone IVRI therapy. CMT before injection and one or two month after injection were shown in figure 2 . 


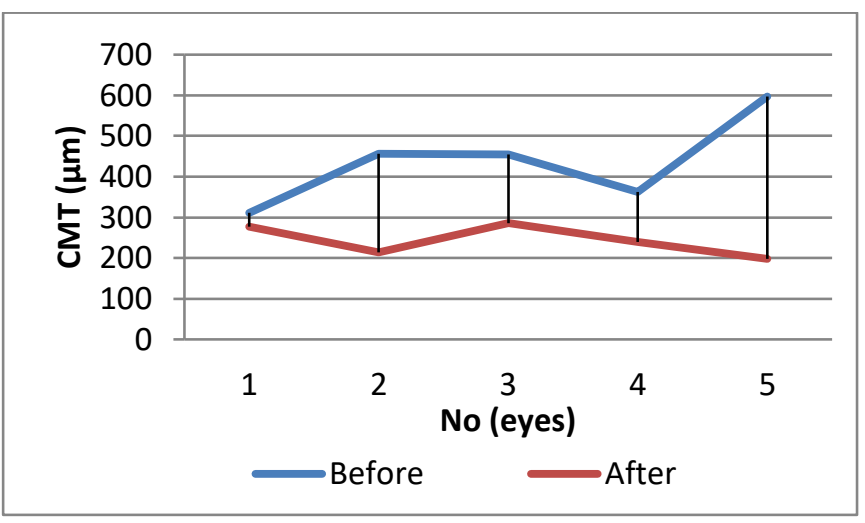

Figure 2. CMT Before and After IVRI

The association between CMT and BCVA as seen from the scatter plot, improvement of BCVA occurred in reduced CMT (figure 3). From the plot, reduced CMT and improvement of BCVA were found after IVRI.

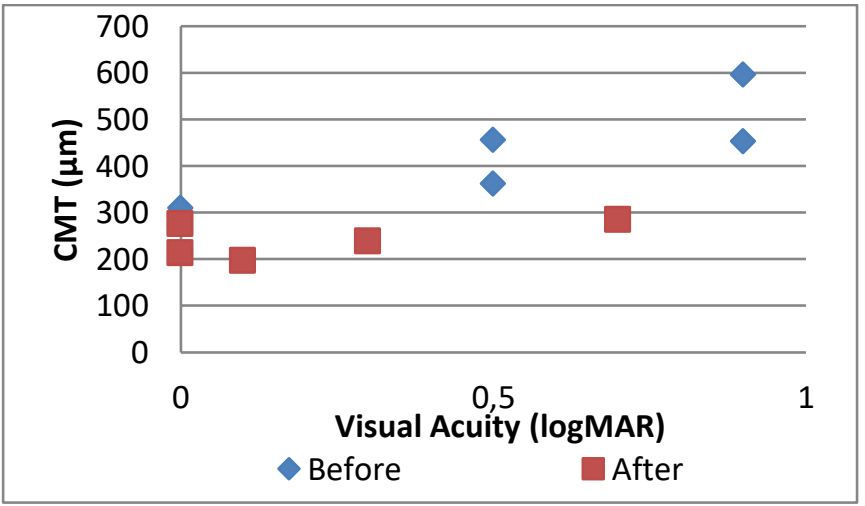

Figure 3. CMT and BCVA Before and After IVRI

Two patients experienced complication at 1 week follow up after injection. Both of them had subconjunctiva bleeding and diminished at one month follow up record.

\section{DISCUSSION}

CSC has become the most common vision threatening disease after age-related macular degeneration (AMD), diabetic retinopathy and retinal vein occlusion. ${ }^{1}$ Men are mostly affected, a six-times higher incidence in men than women and a higher mean age, between 39 and 51 years old, of affected patients. ${ }^{1,3,8}$ This is showed in the result of the study. Mean age was was more than 50 years old compare with the previous research that showed CSC often affect at the young age. ${ }^{1,2,3}$ The older patients show more bilaterality, female prevalence and increased risk of developing choroidal neo-vascularization $(\mathrm{CNV})^{8}$. This maybe the reason more female affected by CSC in the study than male.
CSC presents often as a bilateral condition even though CSC usually reported by patients in one eye due to the presence of a macular SRD. Up to $40 \%$ cases of bilateral CSC had been reported. ${ }^{9}$ But in this study all of the patients had CSC unilaterally

In acute stage of CSC, patients usually complain of blurred vision, central scotoma, metamorphopsia, dyschromatopsia, micropsia, and hypermetropization due to the SRF in the macular area. ${ }^{1,2,3}$ In this study most of the patients complained about metamorphopsia which gradually increased. Other patient also complained the scotoma.

Most acute CSC patients show spontaneous visual recovery within 4 months and this period of time became a consensual definition of acute $\operatorname{CSC}^{2,5}$ Therefore, many clinician considered observation as a first-line approach. Some of this acute CSC, up to $50 \%$ of patients, progresses to chronic stage or recurrent disease within the first year of presentation. This stage is indicated by areas of RPE atrophy and pigmentation in the macular with patients experience subsequent visual loss. When symptoms persist for more than 3 months then active treatment should be initiated and mostly could speeds up visual recovery. Two other condition early treatment is recommended are in cases where rapid recovery of vision for vocational reasons is required and where untreated CSC had previously resulted in a poor visual outcome in the fellow eye. ${ }^{1,25,9}$ Usually, active treatment for CSC is indicated in persistent macular SRD for at least 4 months, history of CSC in the fellow eye with poor visual outcome, history of multiple recurrences, reduced visual acuity, and rapid recovery required. ${ }^{2,10}$ Even though the most suitable treatment and the optimal timing for intervention is not yet concluded.

Active treatment of CSC is intended to induce reattachment of the neurosensory retina, improve or preserve visual acuity, and prevent recurrences. ${ }^{10}$ Oral treatment (carbonic anhydrase inhibitors, beta-blockers, antibiotics, proton pump inhibitors, glucocorticoidreceptor antagonists, anti-platelets, antimetabolites, 5areductase inhibitors, diarylheptanoids), half-dose Verteporfin photodynamic therapy (PDT), laser photocoagulation (green and yellow), and also antiVEGF agents had been estimated as the treatment for CSC. ${ }^{1,2,3,10}$ 
Oral treatment usually given in the observation period waiting for the resolve of the fluid. Studies of laser treatment or subthreshold laser treatment showed varied results due to various laser setting and the area of application of the laser spots. Half dose PDT also showed complete resolution of acute CSC and improvement of $B C V A^{2,3,10}$ but lack of resources to carry out PDT is one of the setback in the use of such therapy. In cases where persistent subretinal fluid exist, it is treated by laser treatment and PDT as the current standart of care, but they are not appropriate in all case and still no consensus of the optimal timing. ${ }^{1,2,3}$ The selection of anti-VEGF therapy rather than half-dose PDT or laser treatment in this study based on the availability of equipment and resources and several studies have shown anti-VEGF therapy is also effective in some cases of CSC both acute and chronic.

Although VEGF was not increased in the aqueous humor and plasma of CSC patients, ${ }^{11}$ anti-VEGF therapy was suggested to reduce the choroidal hyperpermeability. ${ }^{12}$ Intravitreal bevacizumab injection has beneficial effect in visual acuity improvement and SRF reduction without significant complications in case series report. ${ }^{11,13}$ Aflibercept showed moderate effect on fluid and macular thickness reduction in a pilot study of 12 patients with persistent fluid over 6 months. ${ }^{14}$ Ranibizumab showed as an effective treatment on CNV complicating CSC. ${ }^{15}$

Ranibizumab is a small humanized anti-VEGF antibody fragment designed to competitively bind all VEGF isoform. Ranibizumab has smaller molecular weight than bevacizumab and possible deeper penetration into choroidal vascular hyperpermeability lesions in patients with $\operatorname{CSC}^{16}$

The study of efficacy of anti-VEGF drugs (Ranibizumab or Bevacizumab) and compared the results with observational group in acute CSC showed that although BCVA and SRF was not statistically difference between groups, anti-VEGF group had a favorable effect in terms of healthy IS/OS line. ${ }^{17}$ In this study mean time was 1.2 months for the complete resolution of SRF in anti-VEGF group. ${ }^{17}$ In our study we observed the complete SRF resolution in the first and a half months of anti-VEGF injection

Reason of visual acuity worsening is the disruption of the IS/OS junction viewed through OCT. In chronic CSC after resolution of fluid, the foveal thickness is less in affected eyes. Long term atrophy in CSC also contributed in decreasing visual acuity. Those with foveal thinning and atrophy have worse vision. ${ }^{10}$

Our study demonstrated that, IVRI in acute CSC is associated with rapid SRF reduction with a reduction of central macular thickness and increase in visual acuity. Kim et al. reported effectiveness of IVRI for acute CSC (symptomatic for less than 3 months) compared with observation. ${ }^{18}$ Although no statistically significant difference between groups at the sixth month, the observation group required longer time for complete resolution of neurosensory retinal detachment.

Most of the research showed complete resolution of neurosensory retinal detachment in single amount of anti-VEGF injection. In our study showed three out of five patients had complete SRF resolution in one month after single IVRI. Two patients had additional one IVRI to achieved the same result. Bae et all comparing ranibizumab with half PDT with 1 year of follow up. In the ranibizumab group there were $25 \%$ eyes achieved complete resolution of SRF after consecutive IVRI. ${ }^{19}$ Moreover 3 consecutive IVRI showed impressive response in a case of CSC complicated by accumulation of subretinal fibrin. ${ }^{20}$

The anatomic and functional improvement after IVRI suggests a potential role for anti-VEGF agents in the treatment of CSC. Further assessment of intravitreal ranibizumab for CSC in controlled randomized large number of patients with longer follow-up period is required to confirm the efficacy of ranibizumab.

\section{CONCLUSION}

There are visual acuity improvement and central macular thickness reduction in 1 and a half months after IVRI injection. These results strengthen IVRI to be an alternative therapy in CSC.

\section{REFERENCES}

1. Daruich A, Matet A, Dirani A, Bousquet E, Zhao M, et al. Central serous chorioretinopathy: Recent findings and new physiopathology hypothesis. Progress in Retinal and Eye Research 48 (2015) 82118

2. Iaconoa $\mathrm{P}$, Parodi $\mathrm{MB}$, Falcomatà $\mathrm{B}$, Bandello $\mathrm{F}$. Central Serous Chorioretinopathy Treatments: A Mini Review. Ophthalmic Res 2016;55:76-83 
3. Das S, Das D. Central serous chorioretinopathy (CSCR). Sci J Med \& Vis Res Foun 2017;35:10-20

4. Kana $E$, Yucel OE. Recovery of chronic central serous chorioretinopathy with a single dose of intravitreal ranibizumab injection: A case report J. Exp. Clin. Med., 2015; 32(4): 183-186

5. Khan NA, Khan AA, Khan A, Memon Jl. Ranibizumab in the Treatment of Chronic Central Serous Chorio Retinopathy. AdvOphthalmol Vis Syst 2017, 6(5): 00196

6. Fine HF, Ober MD. Current concepts in managing central serous chorioretinopathy. Ophthalmic Surgery, Lasers \& Imaging Retina. 2014;45:9-13

7. Nawaiseh I, Halawa A, Alardah D Intravitrealranibizumab in the management of acute central serous chorioretinopathy. Int J ClinExpOphthalmol. 2017; 1: 049-054

8. Tsai DC, Chen SJ, Huang CC, Chou P, Chung, et al. 2013a. Epidemiology of idiopathic central serous chorioretinopathy in Taiwan, 2001e2006: a population-based study. PLoS One 8, e66858

9. Gackle HC, Lang GE, Freissler, KA, Lang, GK, 1998. Central serous chorioretinopathy. Clinical, fluorescein angiography and demographic aspects. Ophthalmology 95, 529-33

10. Nicholson, Noble J, Forooghian F, Meyerle C. Central Serous Chorioretinopathy: Update on Pathophysiology and Treatment Benjamin SurvOphthalmol. 2013 March ; 58(2): 103-126

11. Lim, J.W., Kim, M.U., Shin, M.C., 2010. Aqueous humor and plasma levels of vascular endothelial growth factor and interleukin-8 in patients with central serous chorioretinopathy. Retina. 30, 14651471.

12. Chung, Y.-R., Seo, E.J., Lew, H.M., Lee, K.H., 2013. Lack of positive effect of intravitreal bevacizumab in central serous chorioretinopathy: meta-analysis and review. Eye (Lond.) 27, 1339-1346

13. E Smretschnig, S Hagen, C Glittenberg, R Rist, I Krebs et al. Intravitreal anti-vascular endothelial growth factor combined with half-fluence photodynamic therapy for choroidal neovascularization in chronic central serous chorioretinopathy. Eye (2016) 30, 805-811

14. Pitcher JD, Witkin AJ, DeCroos FC, et al. A prospective pilot study of intravitreal aflibercept for the treatment of chronic central serous chorioretinopathy: the CONTAIN study. $\mathrm{Br} J$ Ophthalmol 2015;99:848-52

15. Konstantinidis L, Mantel I, Zografos L, Ambresin A, 2010. Intravitreal ranibizumab in the treatment of choroidal neovascularization associated with idiopathic central serous chorioretinopathy. Eur. J. Ophthalmol. 20, 955-8

16. Lantry, L.E., 2007. Ranibizumab, a mAb against VEGF-A for the potential treatment of age-related macular degeneration and other ocular complications. Curr. Opin. Mol. Ther. 9, 592-602

17. Park, S.U., Lee, S.J., Kim, M., 2014. Intravitreal antivascular endothelial growth factor versus observation in acute central serous chorioretinopaty: One-year results. Korean J. Ophthalmol. 28, 306-313.

18. Kim $M$, Lee $S$, Lee $S$. Intravitrealranibizumab for acute central serous chorio-retinopathy. Ophthalmologica 2013; 229:152-157

19. Bae SH, Heo JW, Kim C, Kim TW, Lee JY, Song SJ, Park TK, Moon SW, Chung H: A randomized pilot study of low-fluence photodynamic therapy versus intravitreal ranibizumab for chronic central serous chorioretinopathy. Am J Ophthalmol 2011;152:784-792.e2.

20. Symeonidis C, Kaprinis K, Manthos K, Androudi S, Anastassilakis K, et all. Central Serous chorioretinopathy with subretinal deposition of fibrin-like material ans its prompt response to ranibizumab injections. Case Rep Ophthalmol 2011;2:59-64

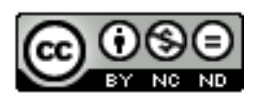

This work licensed under Creative Commons Attribution 